\title{
The Lubricity of Polyvinyl Alcohol and Nano-silica Hydrogel's Scaffold Fabricated by 3D Printing
}

\author{
Zhang Yikun ${ }^{1}$, Wang Hui ${ }^{1}$, Li Xuefeng ${ }^{2,}$ * \\ ${ }^{1}$ Hubei Provincial Key Laboratory of Green Materials for Light Industry, Hubei University of Technology, Wuhan, China \\ ${ }^{2}$ School of Materials and Chemical Engineering, Hubei University of Technology, Wuhan, China
}

\section{Email address:}

li_xf@mail.hbut.edu.cn (Li Xuefeng)

${ }^{*}$ Corresponding author

\section{To cite this article:}

Zhang Yikun, Wang Hui, Li Xuefeng. The Lubricity of Polyvinyl Alcohol and Nano-silica Hydrogel's Scaffold Fabricated by 3D Printing. Science Discovery. Vol. 5, No. 7, 2017, pp. 524-528. doi: 10.11648/j.sd.20170507.19

Received: November 15, 2017; Accepted: November 22, 2017; Published: December 29, 2017

\begin{abstract}
Hydrogel is a macromolecule three-dimensional network made of hydrophilic polymers, which is soft and has good elasticity. Hydrogels have a wide range of potential applications in tissue engineering, drug delivery and biosensors. In this paper can be applied to a biomedical polymer material polyvinyl alcohol as matrix, according to the first forming crosslinking method, using $3 \mathrm{D}$ printing to make $\mathrm{SiO}_{2} / \mathrm{PVA}$ sol forming, and then freeze-melt cross-linking treatment to prepare a physically cross-linked three-dimensional network hydrogel scaffold. The further study found that hydrogel scaffold has high mechanical strength and fine internal structure, the compressive modulus of the hydrogel scaffold can reach more than 2.4 times that of the bulk polyvinyl alcohol hydrogel, and the compression of the scaffold Modulus decreases with increasing porosity of the stent. The tribological properties of the scaffolds show that the scaffold has high lubricity and good stability when rubbed repeatedly, due to the double network hydrogel scaffold has good biological compatibility and adhesion properties, cell culture, cells found on the inner wall of the stent porosity can better growth.
\end{abstract}

Keywords: 3D Printing, Poly (Vinyl Alcohol), Scaffold, Lubricity

\section{D打印聚乙烯醇/纳米氧化硅水凝胶支架润滑性能研究}

\author{
张奕坤 $^{1}$, 王慧 ${ }^{1}$, 李学锋 ${ }^{2 *}$ \\ 1 绿色轻工材料湖北省重点实验室, 湖北工业大学, 武汉, 中国 \\ ${ }^{2}$ 材料与化学工程学院, 湖北工业大学, 武汉, 中国 \\ 邮箱 \\ li_xf@mail.hbut.edu.cn (李学锋)
}

摘要: 水凝胶是由亲水性聚合物构成的高分子三维网络, 柔软并有较好的弹性, 水凝胶在组织工程、药物缓释、 生物传感器领域有着广泛的潜在应用。本文采用可应用于生物医学的高分子材料聚乙烯醇为基体, 根据先成型 后交联的方法, 利用3D打印技术使聚乙烯醇与二氧化硅的溶胶成型, 将所得的水凝胶支架经冷冻-熔融的交联处 理, 制备出物理交联三维网络状水凝胶支架。经进一步研究发现, 该水凝胶支架具有较高的力学强度及精细的 内部结构, 其水凝胶支架的压缩模量可达到块体聚乙烯醇水凝胶的 2.4 倍以上, 并且支架的压缩模量随支架孔隙 率的增大而减小。通过摩擦性能的测试发现, 该支架拥有着较高的润滑性能, 并且重复摩擦时具有良好的稳定 性。由于该水凝胶支架具有较好的生物相容性和粘附性能, 对其进行细胞培养, 发现细胞在支架孔隙内壁能够 较好的生长。 
关键词: 3D打印, 聚乙烯醇, 支架, 润滑性

\section{1. 引言}

水凝胶由于在力学性能和自修复性能上的巨大潜力 使它在仿生材料[1]、人造胰腺[2]、人造血管[3-4]、人工 软骨 [5-8]这些组织工程领域有巨大应用前景。水凝胶具有 良好的力学性能, 可以保护水凝胶在大形变下不会发生损 坏，同时优良的自修复性能使已经破坏的水凝胶能够自主 修复材料破损的结构。因此，制备出同时具有优秀的力学 性能以及自修复性能的水凝胶迫在眉睫[9]。通过构建新型 网络结构以及能量耗散方式能够提高水凝胶的强度和韧 性, 例如:双网络(DN)水凝胶、滑动环水凝胶、三组分共聚 水凝胶、疏水水凝胶、大分子微球复合 $(\mathrm{MMC})$ 水凝胶。为 了能够承受巨大的内部和外部的载荷, 生物软骨组织通常 具有高断裂韧性。而由于衰老、外伤等原因人类关节软骨 又常易损坏, 同时, 软骨组织因再生能力弱, 其修复仍是 当今世界一大难题[10]。正因为如此, 使得更多的研究人 员去探索类似生物组织韧性的凝胶体来替代生物组织。尽 管目前在韧性水凝胶的研究方面取得了一些成功, 但这些 合成水凝胶通常涉及到有毒化学品及艰难的合成工艺, 限 制了水凝胶在封装细胞方面的应用 [11]。目前, 3D打印做 为一种使用工具已经出现, 目的在于设计复杂精细的凝胶 组织工程支架, 这种技术在设计一系列凝胶支架结构方面 具有很大的潜在应用前景。

根据先前报道，目前有很多材料制备的支架适用于组 织工程, 而最受欢迎的材料类型通常为天然的和合成的高 分子。本研究中选择了生物相容性材料聚乙烯醇来作为制 备水凝胶支架的材料, 通过3D打印方法制备聚乙烯醇/纳 米氧化硅复合水凝胶支架。

\section{2. 实验材料及方法}

\section{1. 实验材料}

聚乙烯醇（PVA，分析纯，聚合度 $1750 \pm 50$, 国药集 团化学试剂有限公司), 气相二氧化硅（粒径 $7 \sim 40 \mathrm{~nm}$, 阿拉丁试剂（上海）有限公司）。

\section{2. 聚乙烯醇/纳米氧化硅复合溶胶的制备}

按质量浓度为 $10 \%$ 的PVA加入去离子水中搅拌分散, 于 $95^{\circ} \mathrm{C}$ 恒温水浴锅中冷凝回流 $2 \mathrm{~h}$, 至PVA完全溶解, 得 到PVA溶液; 将所得的混合溶液于 $95^{\circ} \mathrm{C}$ 真空干燥箱内脱气 $0.5 \mathrm{~h}$, 脱气完毕, 将混合溶液缓慢的加入无机粉体气相二 氧化硅 $\left(\mathrm{SiO}_{2}\right)$ 中, 并摚拌均匀, 使混合溶液与气相二氧 化硅的质量比为 $1: 1$, 得到具有PVA复合溶胶。

\subsubsection{D打印聚乙烯醇/海藻酸盐水凝胶支架}

如图1所示利用机器人点胶机驱动医用注射器连接精 细针管或移液管滴头, 通过调节所述点胶机XY轴平台的 移动速率、Z轴步进高度、点胶机的挤出体积流率这些技
术参数, 挤出配制好的溶胶, 3D打印成型得到溶胶支架样 品; 将打印出的溶胶支架置于 $-20^{\circ} \mathrm{C} \sim-40^{\circ} \mathrm{C}$ 冷冻室冷冻 20 24小时, 使聚合物PVA引发聚合并充分交联成网络得 到预成型的凝胶支架, 取出室温解冻3 4小时。

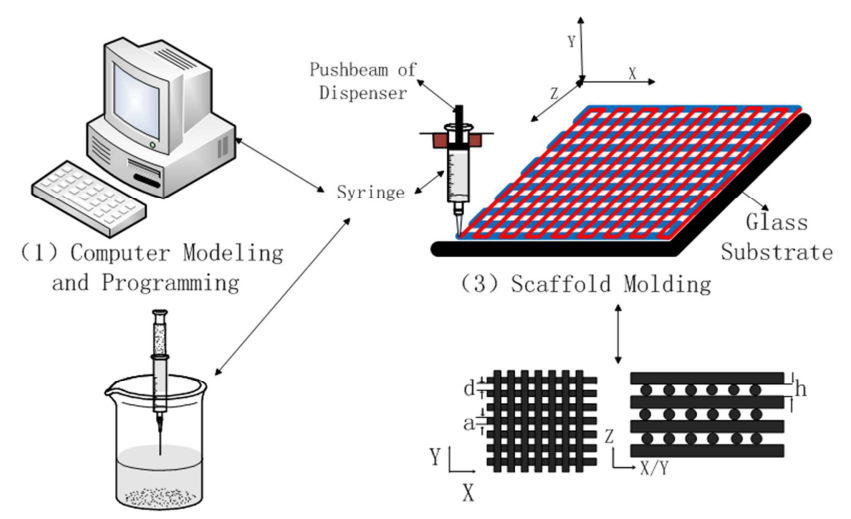

(2) Material Preparation

(4) After-treatment

图1 $\mathrm{SiO}_{2} / \mathrm{PVA}$ 水凝胶3D成型过程示意图。

通过调节挤出速率制备了不同孔隙率的 $\mathrm{SiO}_{2} / \mathrm{PVA}$ 水 凝胶支架, 分别记为 $\mathrm{SiO}_{2} / \mathrm{PVA}$ 水凝胶支架 $1 、 2$ 和 3 。采用 同等配方条件注入两平行玻璃板模具中再冷冻-熔融的交 联得到对比块体 $\mathrm{SiO}_{2} / \mathrm{PVA}$ 水凝胶样品。将成型的 $\mathrm{SiO}_{2} / \mathrm{PVA}$ 水凝胶支架和 $\mathrm{SiO}_{2} / \mathrm{PVA}$ 块体水凝胶置于去离子 水中浸泡至少三天, 每天换水至少两次, 除去未反应完的 单体及小分子。

\section{4. 孔隙率测试}

待水凝胶支架在去离子水中溶胀平衡后取出, 并用滤 纸擦干支架表面的去离子水, 用电子游标卡尺量出水凝胶 支架的直径 $d$ 和厚度 $h$, 再将其浸入去离子水中, 测出排出 水体积 $V$ 。多孔材料的孔隙率由孔隙所占体积 $V_{1}$ 与多孔总 体积 $V_{2}$ 的比率来计算, 以百分数表示, 由式 (1) 计算, 经过换算孔隙率 $P$ 可由式 (2) 计算得到。每组实验对水凝 胶支架测试3-4次, 最后结果取测试结果的平均值。

$$
\begin{gathered}
P=\frac{V_{1}}{V_{2}} \\
P=\frac{\pi\left(\frac{d}{2}\right)^{2} h-V}{\pi\left(\frac{d}{2}\right)^{2} h} \times 100 \%
\end{gathered}
$$

\section{5. 机械性能测试}

\subsection{1. 压缩性能测试}

利用电子万能拉力试验机 (CMT4000, MTS系统 (中 国) 有限公司) 测试 $\mathrm{SiO}_{2} / \mathrm{PVA}$ 水凝胶支架压缩性能, 拉伸 
速率为 $0.3 \mathrm{~mm} / \mathrm{min}$, 所测试支架为圆柱体, 直径为 $13 \mathrm{~mm}$, 厚 $3 \mathrm{~mm}$ 。

\subsection{2. 摩擦性能测试}

采用平板流变仪 (DHR-2, 美国TA Instruments公司) 测试 $\mathrm{SiO}_{2} / \mathrm{PVA}$ 双网络水凝胶支架和PVA、SA单网络水凝 胶支架与玻璃基板之间的摩擦行为, 采用连续摩擦和阶梯 摩擦两种不同的模式进行测试。制备的凝胶支架作为软基 板, 支架粘附在流变仪上基板, 调节流变仪平板 gap值, 使得支架承受的载荷达到 $3 \mathrm{KPa}$ 。连续 $\omega$ 模式和阶梯模式下 的剪切速率范围为 $10^{-3} \sim 10^{2} \mathrm{rad} / \mathrm{s}$, 阶梯模式下, 同一样本 需做3次重复性测试, 根据公式 (3) 算出摩擦力 $F$, 并由 公式 (4) 算出滑动速率 $V$ 。

$$
\begin{gathered}
F=\frac{4 T}{3 R} \\
V=\omega \cdot R
\end{gathered}
$$

式中: $\mathrm{R}$ 一试样的半径; $\omega$ 一角速度。

2.6. 生物相容性测试

\subsection{1 . 消毒灭菌}

将水凝胶支架置于 $100^{\circ} \mathrm{C}$ 的高温蒸汽中灭菌 1 小时, 然 后取出水凝胶支架, 并将水凝胶支架置于紫外光等下消毒 3-4小时。

\subsection{2. 细胞接种}

将细胞培养液加入到人体正常肝细胞L-02置于培养 瓶中, 细胞贴壁大量生长时将培养液移去, 接着用 $2 \mathrm{ml}$ 的 PBS缓冲溶液冲刷细胞, 加入 $2 \mathrm{ml}$ 的 $0.25 \%$ 的胰蛋白酶使细 胞从瓶壁上脱离下来, 将含有人体正常肝细胞L-02的培养 液转移在无菌离心管中离心 $5 \mathrm{~min}$, 得到细胞悬浮液。

在无菌工作台上, 将水凝胶支架放进 24 孔 $2 \times 10^{4} / \mathrm{m}$ 培 养孔板中, 然后将 $300 \mu \mathrm{L}$ 培养液置于孔板中, 孔板盖盖上 并留缝隙, 将培养孔板放入 $37^{\circ} \mathrm{C}, 5 \% \mathrm{CO}_{2}$ 浓度, $100 \%$ 湿 度的恒温、恒湿的 $\mathrm{CO}_{2}$ 培养箱中。每24小时, 除去每个孔 板内的培养液, 并加入新的细胞培养液, 使正常人体肝细 胞L-02细接种在水凝胶支架上。

\section{3. 结果与讨论}

\section{1. $\mathrm{SiO}_{2} / \mathrm{PVA}$ 水凝胶支架的形貌测试}

通过先成型后交联两步法制备了不同孔隙率的 $\mathrm{SiO}_{2} / \mathrm{PVA}$ 复合水凝胶支架, 通过光学显微镜对不同孔隙 率水凝胶支架的正面和断面静置观察。如图2所示, 图a 为 $3 \mathrm{D}$ 水凝胶支架的俯视图, 图b、c、 $d$ 为 $\mathrm{SiO}_{2} / \mathrm{PVA}$ 复合 水凝胶支架的断面图, 从图中可以看出, $\mathrm{SiO}_{2} / \mathrm{PVA}$ 水凝 胶支架的纤维丝的直径约为 $500 \mu \mathrm{m}$, 并且支架具有清晰 的孔径结构。通过计算可以得出 $\mathrm{SiO}_{2} / \mathrm{PVA}$ 水凝胶支架 1 、 2 和 3 的孔隙率分别为 $23.2 \% 、 32.7 \%$ 和 $42.3 \%$ 。
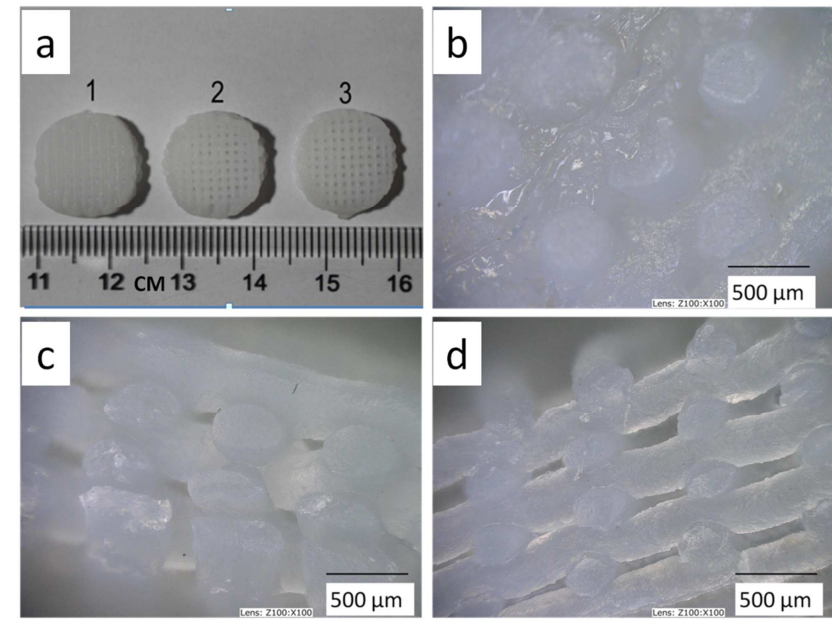

图2 不同孔隙率的 $\mathrm{SiO}_{2} / \mathrm{PVA}$ 水凝胶支架的（a）俯视图和（b）断面图， $23.2 \%$ 、（c） $32.7 \%$ 、（d） $42.3 \%$ 。

\section{2. 压缩性能的测试}

用万能电子拉力试验机测得不同孔隙率 $\mathrm{SiO}_{2} / \mathrm{PVA}$ 水 凝胶支架的压缩模量。 $\mathrm{SiO}_{2} / \mathrm{PVA}$ 水凝胶支架在压缩过程 中, 在 $40 \%$ 压缩应变范围内支架完好没有出现破损, $\mathrm{SiO}_{2} / \mathrm{PVA}$ 水凝胶支架表现出良好的韧性。对压缩应变曲 线进行线性拟合, 可以得到 $10 \%$ 之前的压缩应力-应变曲 线, 从而得出水凝胶支架的压缩模量。

表 $1 \mathrm{SiO}_{2} / \mathrm{PVA}$ 水凝胶支架和块体 $\mathrm{SiO}_{2} / \mathrm{PVA}$ 水凝胶的压缩模量。

\begin{tabular}{ll}
\hline 样品 & 压缩模量（KPa） \\
\hline Bulk PVA hydrogel & 12.0 \\
Scaffold(23.2\%) & 39.2 \\
Scaffold $(32.7 \%)$ & 38.2 \\
Scaffold $(42.3 \%)$ & 36.8 \\
Bulk SiO 2 PVA hydrogel & 62.0 \\
\hline
\end{tabular}

$\mathrm{SiO}_{2} / \mathrm{PVA}$ 水凝胶支架的压缩模量随 $\mathrm{SiO}_{2} / \mathrm{PVA}$ 水凝胶 支架的孔隙率的增加而呈下降的趋势, 其中块状 $\mathrm{SiO}_{2} / \mathrm{PVA}$ 的压缩模量最大 $(62 \mathrm{KPa})$, 并且水凝胶支架的压缩模量 比同样配比的块体水凝胶的压缩模量低。这是由于块体水 凝胶的宏观网络较水凝胶支架更为致密, 并且水凝胶支架 的木堆结构能够耗散部分外力的作用, 使水凝胶支架表现 出比块体水凝胶较好的压缩回弹性能。

\section{3. 摩擦性能的测试}

图3、图4分别为块体水凝胶与不同孔隙率的 $\mathrm{SiO}_{2} / \mathrm{PVA}$ 水凝胶支架摩擦力随载荷的变化图。 $\mathrm{SiO}_{2} / \mathrm{PVA}$ 水凝胶支架 与块体 $\mathrm{SiO}_{2} / \mathrm{PVA}$ 水凝胶的摩擦力均随所受载荷的增大而增 大, 且两者的摩擦行为基本一致, 当摩擦速率较低时, 样 品与基板间的摩擦力均随滑动速率的增大而增大; 增大滑 动速率, 当达到粘弹-流体润滑转变速率时, 摩擦力出现峰 值; 随后摩擦力随滑动速率的增大而减小; 随着滑动速率 的继续增大, 摩擦力又呈现随滑动速率的增大而继续增大 的趋势。低速 $\left(10^{-6} \sim 10^{-3} \mathrm{~m} / \mathrm{s}\right)$ 时, $\mathrm{SiO}_{2} / \mathrm{PVA}$ 水凝胶支架的 摩擦力较 $\mathrm{SiO}_{2} / \mathrm{PVA}$ 水凝胶稍高, $\mathrm{SiO}_{2} / \mathrm{PVA}$ 水凝胶支架的摩 擦力随孔隙率的增加稍有降低; 高速 $\left(10^{-2} \sim 1 \mathrm{~m} / \mathrm{s}\right)$ 时, 孔 隙率对 $\mathrm{SiO}_{2} / \mathrm{PVA}$ 支架摩擦力影响不明显, $\mathrm{SiO}_{2} / \mathrm{PVA}$ 水凝胶 
支架与 $\mathrm{SiO}_{2} / \mathrm{PVA}$ 块状水凝胶摩擦力相当, 均约为 $0.02 \mathrm{~N}$ 。 $\mathrm{SiO}_{2} / \mathrm{PVA}$ 水凝胶支架仍保持 $\mathrm{SiO}_{2} / \mathrm{PVA}$ 水凝胶的较高润滑
性能，并且具有较高的重复摩擦稳定性，这与支架结构能 稳定地保持润滑水层相关。

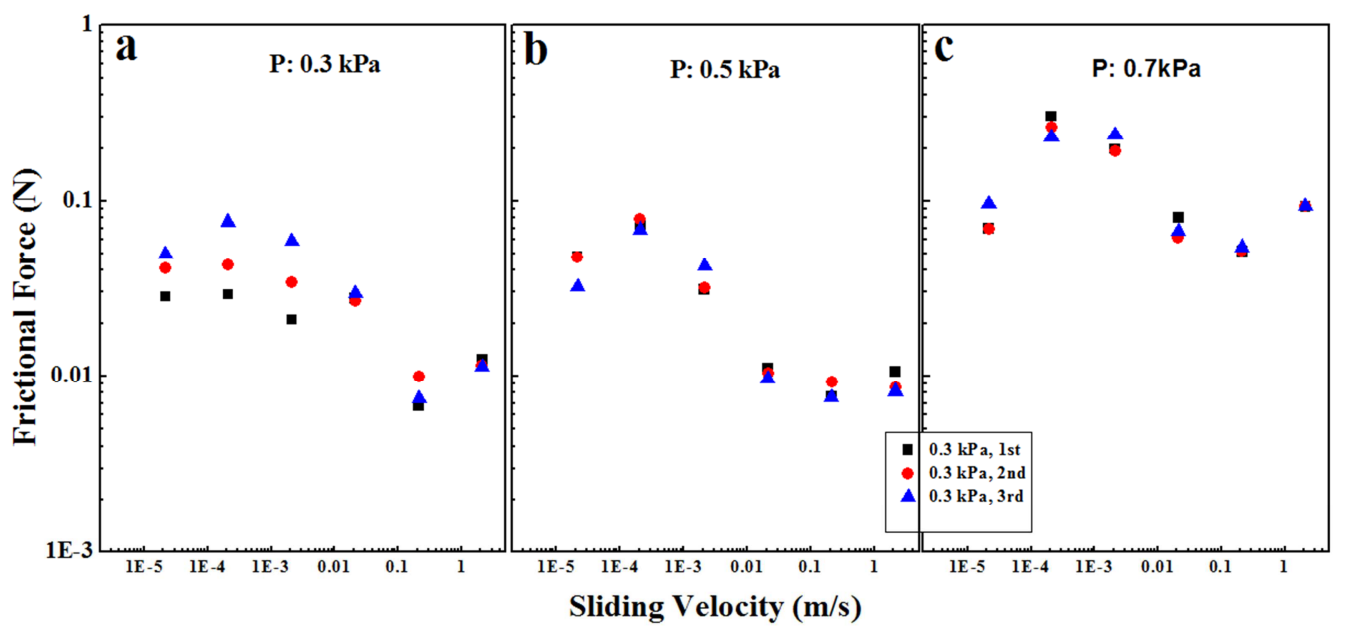

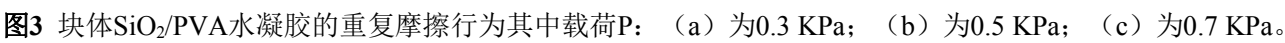

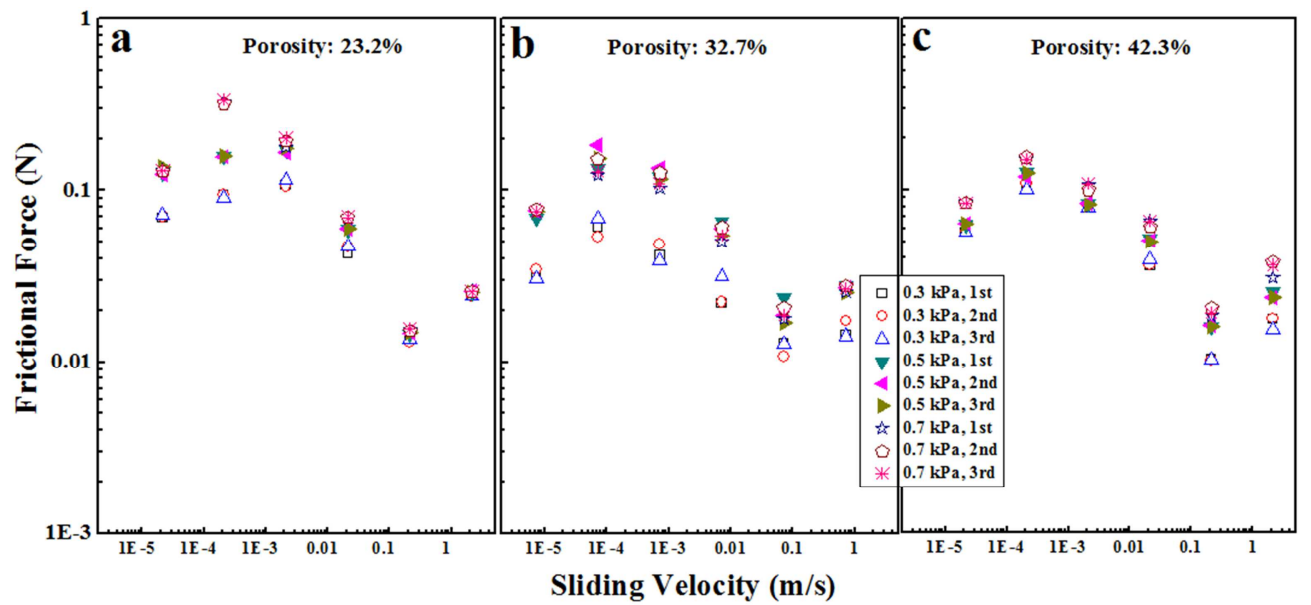

图4 不同孔隙率的 $\mathrm{SiO}_{2} / \mathrm{PVA}$ 水凝胶支架的重复摩擦行为其中孔隙率：（a）为 $23.2 \% ； （ b ）$ 为 $32.7 \%$; (c) 为 $42.3 \%$ 。

根据研究结果表明, 产生如图5所示摩擦机理。在施 加小载荷时，块体水凝胶因表面光滑，会与玻璃基板产生 较强的吸附作用, 因此与基板接触时会产生捕获水, 且在 低速率时，捕获水会在块体水凝胶与基板之间随机移动， 导致块体水凝胶的重复摩擦性较差（图5-a）。随着摩擦 速率增加, 捕获水会在水凝胶与基板之间形成一层薄的水
润滑层, 使得高速区摩擦行为重复性较好。随着载荷增加, 块体水凝胶与基板之间的捕获水逐渐被挤压出去, 低速率 摩擦时, 水凝胶与基板间的自由移动的捕获水减少，重复 摩擦性提高。水凝胶支架由于内部贯通的木堆结构, 使其 在与基板接触时不易产生捕获水, 却能保持稳定的水润滑 层（图5-b），因此水凝胶支架有较好重复摩擦性。

$$
\text { Before contact } P \text {, }
$$
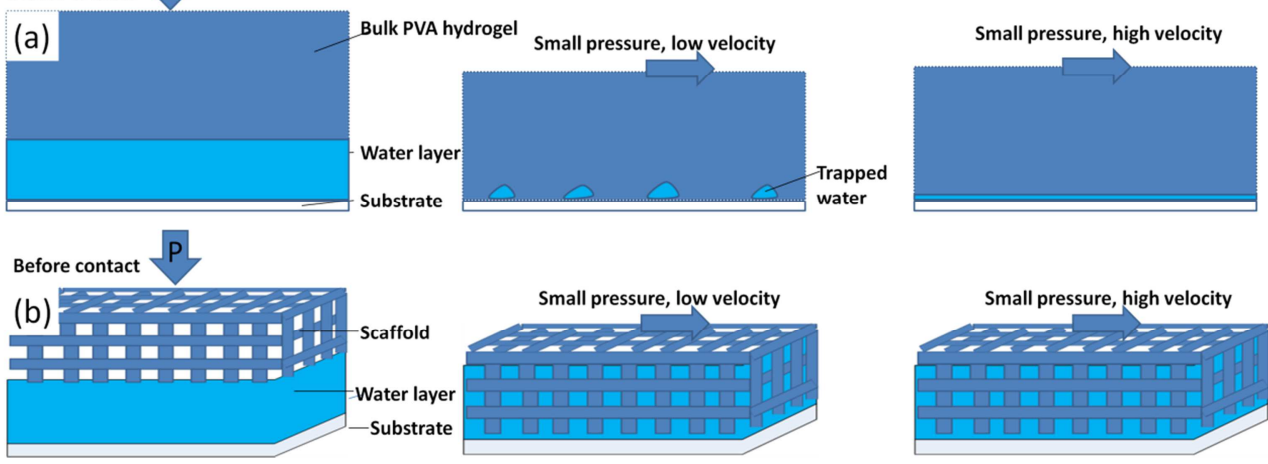

Small pressure, low velocity
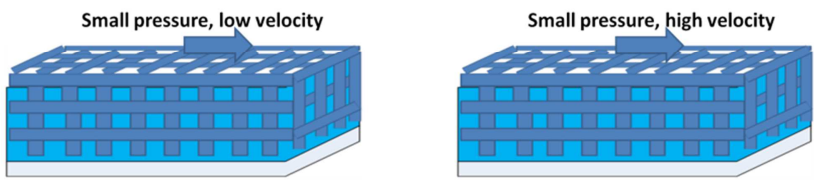

图5 块体 $\mathrm{SiO}_{2} / \mathrm{PVA}$ 水凝胶（a）与 $\mathrm{SiO}_{2} / \mathrm{PVA}$ 水凝胶支架（b）在基板表面不同接触示意图。 


\section{4. $\mathrm{SiO}_{2} / \mathrm{PVA}$ 水凝胶支架的生物相容性研究}

将人体肝细胞L-02接种在制作好的多孔水凝胶生物 支架上, 对肝细胞进行静态观察, 将细胞培养基板放置在 倒置显微镜下观察肝细胞的生长形态。如图6所示, 是人 体肝细胞 L-02在 $\mathrm{SiO}_{2} / \mathrm{PVA}$ 水凝胶支架上的生长情况, 可以 看出随着培养天数增加, 细胞生殖状况越好, 细胞数量随 之增多。由此可以看出 $\mathrm{SiO}_{2} / \mathrm{PVA}$ 水凝胶支架具有良好的生 物相容性。
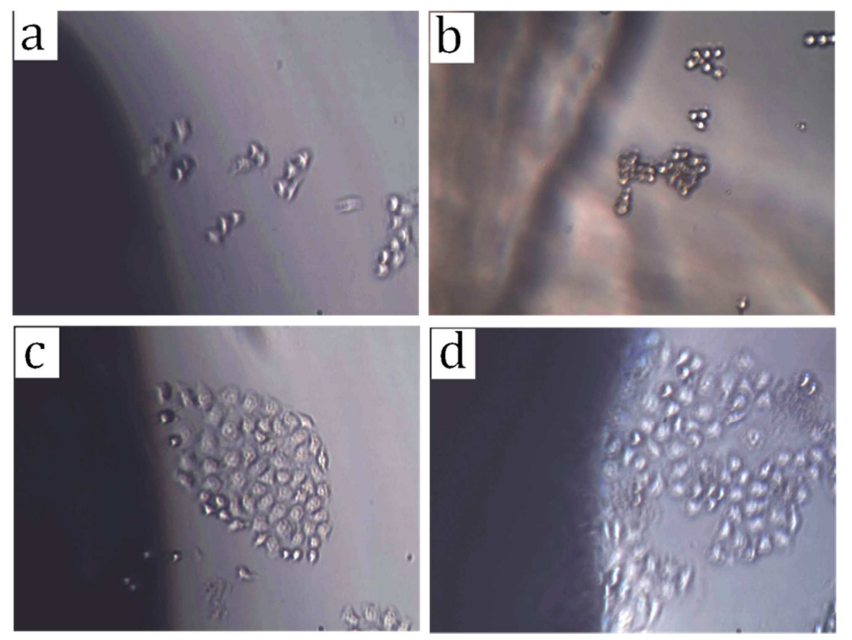

图6 人体肝细胞 $\mathrm{L}-02$ 在 $\mathrm{SiO}_{2} / \mathrm{PVA}$ 水凝胶支架上的生长情况。其中 (a) 第 1 天;（b）第 3 天; (c) 第5天; (d) 第7天。

\section{4. 结论}

$\mathrm{SiO}_{2} / \mathrm{PVA}$ 水凝胶支架通过基于3D打印挤出制造。该 支架是有较高孔隙率的木垛结构。其水凝胶支架的压缩模 量可达到块体聚乙烯醇水凝胶的 2.4 倍以上, 并且支架的 压

缩模量随支架孔隙率的增大而减小。 $\mathrm{SiO}_{2} / \mathrm{PVA}$ 水凝胶 支架仍保持 $\mathrm{SiO}_{2} / \mathrm{PVA}$ 水凝胶的较高润滑性能, 并且具有较 高的重复摩擦稳定性, 这与支架结构能稳定地保持润滑水 层相关, 此外该水凝胶支架有利于细胞的生长, 具有一定 的生物相容性。

除了具有高弹性和良好的摩擦行为重现性以外, 通过 $3 \mathrm{D}$ 打印制备的水凝胶支架具有合成简单, 生物相容性好等 优点。这些理想的特征使得这种材料能够培养一些组织并 重建器官。为水凝胶在生物领域的应用打下了良好的材料 基础, 有着广阔的研究前景。

\section{致谢}

感谢国家自然科学基金委（51605065）和湖北省轻工 绿色材料重点实验室开放基金（201611A04）的支持。

\section{参考文献}

[1] 乔斿, 郑裕东, 李佳琪等. 纳米细菌纤维素/聚乙烯醇复合 水凝胶在模拟体液中的疲劳行为 $[\mathrm{J}]$. 复合材料学报, 2015,32(5):1271-1278。

[2] T H Young, N K Yao, R F Chang, et al, "Evaluation of asymmetric poly (vinyl alcohol) membranes for ues in artifical islets” J. Biomaterials, vol. 17(22), pp. 2139-2145, 1996.

[3] M Oka. "Biomechanics and repair of articular cartilage" J. Journal of Orthopaedic Science, vol. 6(5), pp. 448-456, 2001.

[4] M Oka, Y S Chang, Nakamura T, et al, "Synthetic osteochondral replacement of femoral articular surface" J. Journal of Bone \& Joint Surgery, vol. 79(6), pp 1003-1007, 1997.

[5] M Oka, T Noguchi, P Kumar, et al, "Development of an artificial articular cartilage" J. Clinical Materials, vol. 6(4), pp 361-381, 1990.

[6] T Noguchi, T Yamamuro, M Oka, et al, "Poly (vinyl alcohol) hydrogel as an artificial articular cartilage: evaluation of biocompatibility" J. Journal of Applied Biomaterials, vol. 2(2), pp 101-107, 1991.

[7] W Szkowski, D N Ku, H Bersee, et al, "An elastic material for cartilage replacement in an arthritic shoulder joint" J. Biomaterials, vol. 27(8), pp 1534-1541, 2006.

[8] 李坚, 李学锋, 龙世军等. $3 \mathrm{D}$ 成型 $\mathrm{SiO}_{2} / \mathrm{PVA}$ 水凝胶支架的 摩擦行为 [J]. 复合材料学报, 2016,33(11):2412-2418。

[9] N A Peppas, Y Huang, M Torres Lugo, et al, "Physicochemical foundations and structural design of hydrogels in medicine and biology" J. Annual Review of Biomedical Engineering, vol. 2(1), pp 9-29, 2000.

[10] 李坚, 李学锋, 龙世军等. 3D成型聚乙烯醇水凝胶支架的 摩擦行为 $[\mathrm{J}]$. 复合材料学报, 2016:0-0。

[11] S E Bakarich, P Balding, R Gorkin, et al, "Printed ionic-covalent entanglement hydrogels from carrageenan and an epoxy amine" J. Rsc Advances, vol. 4(72), pp 38088-38092, 2014. 